\title{
TRANSICIONES JUVENILES DE MIGRANTES PARAGUAYOS/AS EN LA ARGENTINA: CONDICIONES DE VIDA Y VIGENCIA DE LAS REDES
}

\author{
ANA MIRANDA* \\ MARÍA CRISTINA CRAVINO ${ }^{* *}$ \\ SANTIAGO MARTÍ GARRO ${ }^{* * *}$
}

\begin{abstract}
RESUMEN
Las migraciones juveniles ocupan un lugar central en la agenda pública y social de América Latina. Su relevancia tiene relación con la envergadura que adquiere la movilidad de los jóvenes y sus grupos familiares, especialmente en los países de la Región Andina y del Caribe. Las migraciones juveniles están fuertemente imbricadas con los procesos de transformación social que signan nuestros días, tales como la globalización, los cambios en el rol de la mujer y los grupos familiares, la conectividad, la reversibilidad, entre otros. Así como también, con tendencias de más larga data, como la reproducción de las desigualdades de clase, patrones tradicionales de género y discriminación étnica, los cuales se materializan en la continuidad de fenómenos de integración - laboral/social - segmentada de los y las jóvenes migrantes en las sociedades de destino. En este contexto, el texto presenta los primeros resultados de una investigación realizada sobre las temáticas de juventud y género entre jóvenes involucrados/as en procesos migratorios entre Paraguay y Argentina. El estudio fue realizado por un equipo binacional integrado por el Viceministerio de la Juventud de Paraguay y el Programa de Investigaciones de Juventud de la FLACSO Argentina, y que contó con el apoyo y financiamiento de ONU Mujeres.
\end{abstract}

PALABRAS CLAVE: JUVENTUD, MIGRACIÓN, GÉNERO

* Doctora en Ciencias Sociales de la FLACSO Argentina. Investigadora adjunta del Conicet y Coordinadora Académica del Programa de Investigaciones de Juventud de la FLACSO, sede argentina.

E-Mail: amiranda@flacso.org.ar.

** Doctora en Antropología y Magíster en Administración Pública. Investigadora docente de la Universidad Nacional General Sarmiento (UNGS). E-Mail: ccravino@ungs.edu.ar.

*** Licenciado en Sociología de la Universidad Nacional de Buenos Aires y maestrando en Demografía Social de la Universidad Nacional de Luján. Forma parte del Programa de Juventud de la FLACSO.

E-Mail: santiagomg@gmail.com.

Una primera versión del texto fue presentada en el XXX Congreso de la Asociación de Estudios Latinoamericanos (LASA), de mayo de 2012. 


\title{
TRANSIÇÕES JUVENIS DE MIGRANTES PARAGUAIOS/AS NA ARGENTINA: CONDIÇÕES DE VIDA E DURAÇÃO DAS REDES
}

\begin{abstract}
RESUMO
As migrações juvenis são centrais para a agenda pública e social na América Latina. Sua relevância está relacionada com a importância da mobilidade dos jovens e de seus grupos familiares, em especial nos países da Região Andina e do Caribe. As migrações juvenis estão fortemente associadas com os processos de transformação social que marcam nossos dias, tais como a globalização, as mudanças do papel das mulheres e grupos familiares, conectividade, reversibilidade, entre outros, e também tendências mais consolidadas, como a reprodução das desigualdades de classe, padrões tradicionais de gênero e discriminação étnica. Esses processos se materializam na continuidade dos fenômenos de integração trabalhista e social dos/as jovens migrantes nas sociedades de destino. Neste contexto, o artigo apresenta os primeiros resultados de uma pesquisa sobre os temas de juventude e gênero entre jovens em processos de migração entre Paraguai e Argentina. $\mathrm{O}$ estudo foi realizado por uma equipe binacional composta pelo Vice-Ministério da Juventude do Paraguai e pelo Programa de Pesquisa da Juventude de FLACSO Argentina, tendo recebido apoio e financiamento da ONU Mulheres.
\end{abstract}

PALAVRAS CHAVE: JUVENTUDE, MIGRAÇÃO, GÊNERO

\section{JUVENILE MIGRATION FROM PARAGUAY TO ARGENTINA: LIVING CONDITIONS AND DURATION OF NETWORKS}

\begin{abstract}
Juvenile migration are central to the social and public agenda in Latin America. Its relevance is related to the extent that acquires mobility of young people and their family groups, especially in the countries of the Andean and Caribbean region. Juvenile migration are strongly intertwined with the social transformation processes that mark our days, such as globalization, the changing role of women and family groups, connectivity, reversibility, among others. Just as well, with more long-standing trends, as the reproduction of class inequalities, traditional patterns of gender and ethnic discrimination, which are embodied in the continuity of phenomena-labor integration, social-segment of young migrants in receiving societies. This article presents the first results of a research on the themes of youth and gender among youth involved/as in migration processes between Paraguay and Argentina. The study was conducted by a bi-national team, composed by the Vice-Ministry of Youth Paraguay and Youth Research Program at FLACSO Argentina, and received support and funding from UN Women.
\end{abstract}




\section{INTRODUCCIÓN}

LAS MIGRACIONES JUVENILES OCUPAN un lugar central en la agenda pública y social de América Latina. Su relevancia tiene relación con la envergadura que adquiere la movilidad de los jóvenes y sus grupos familiares, especialmente en los países de la Región Andina y del Caribe. Año tras año miles de jóvenes se trasladan hacia otras latitudes en busca de oportunidades laborales, educativas, nuevos estilos de vida y consumo cultural o conviven con la migración de algún miembro de su grupo familiar. Enfrentando, en ambos casos, los desafíos que plantea la movilidad nacional y transnacional durante la transición hacia la adultez.

Las migraciones juveniles están fuertemente imbricadas con procesos de cambio y transformación social que signan nuestros días, como la globalización, las transformaciones en el rol de la mujer y los grupos familiares, la conectividad, la reversibilidad, entre otros. En efecto, no se puede pensar hoy la migración sin hacer mención a las redes sociales, los grupos familiares trasnacionales, la ruptura de la linealidad y la homogeneidad en los tránsitos a la vida adulta (Molgat, 2008). Las migraciones de los/as jóvenes latinoamericanos/as expresan, además, tendencias de más larga data, como la reproducción de las desigualdades de clase, patrones tradicionales de género y discriminación étnica, los cuales se materializan en la continuidad de fenómenos de integración - laboral y socialmente - segmentada de los migrantes en las sociedades de destino (Portes, 2001).

En la intersección entre la movilidad trasnacional y la condición juvenil tiene lugar una dinámica de cambio y conservación social, en donde se cruza e interpela la movilidad individual y familiar de los sujetos (García Borrego, S/F). En esta dirección y frente al tema que nos convoca, es preciso advertir que hace algunos años se hacía difícil pensar la existencia de «jóvenes migrantes», ya que la condición juvenil era privilegio de individuos - generalmente hombres - de grupos socialmente aventajados (Miranda, 2007), los cuales en algunos casos experimentaban la movilidad territorial en calidad de «estudiantes», patrocinados siempre por su red familiar. Mientras que aquellos que hoy denominamos «jóvenes migrantes» se incorporaban a la actividad laboral en las sociedades de destino o permanecían en sus hogares de origen asumiendo las tareas domésticas desde edades tempranas.

En nuestros días, la desigualdad en las formas de transitar la juventud continúa reflejando la distribución de oportunidades y accesos 
de la estructura social. Aquellos que provienen de familias de mayores ingresos tienen a disposición opciones educativas en donde la movilidad ha adquirido un carácter novedoso debido a los procesos de globalización y la mayor conectividad, abriendo posibilidades y extendiendo significativamente el período de la juventud. Por otra parte, aquellos que nacen en grupos familiares de menor poder económico se debaten entre el empleo precario y la inactividad. Frente a lo cual la migración se presenta como una alternativa en busca de «progreso», ya que la debilidad de las opciones laborales en los lugares de origen es el principal detonante de los procesos de migración internacional (Sanchís y Rodríguez Enríquez, 2011).

En este contexto interpretativo, durante el año 2011 se realizó una investigación sobre las temáticas de juventud y género entre jóvenes involucrados/as en procesos migratorios entre Paraguay y Argentina. El estudio fue realizado por un equipo binacional integrado por el Viceministerio de la Juventud de Paraguay y el Programa de Investigaciones de Juventud de FLACSO Argentina, y contó con el apoyo y financiamiento de ONU Mujeres.

La investigación adoptó una estrategia metodológica de carácter exploratorio, que se plasmó a partir de la consulta de estudios, procesamiento de información estadística secundaria y recolección de información primaria a partir de entrevistas en profundidad con jóvenes que participan, participaron o se encuentran afectados/as por la migración. El objetivo central fue brindar relevancia a un colectivo juvenil que se encuentra invisibilizado - y muchas veces denostado- en la opinión pública en ambos países, haciendo fundamental hincapié en la intersección entre juventud, migración y género en el corredor migratorio paraguayo-argentino. A continuación se presenta una selección de los principales hallazgos del estudio.

\section{EL CORREDOR MIGRATORIO PARAGUAYO-ARGENTINO}

Producto de vaivenes socioeconómicos así como de los violentos y repetidos avatares políticos que atravesó, la emigración en Paraguay ha sido un fenómeno constante a lo largo de la historia y una marca característica del país aún en el contexto latinoamericano. Ya desde las últimas décadas del siglo diecinueve la privatización de la tierra impulsó la emigración de grandes contingentes poblacionales cuyo modo de vida dependía de la agricultura, los cuales se dirigieron primero hacia otras zonas del Paraguay y posteriormente a los países 
vecinos. Ese drenaje poblacional continuó a lo largo del siglo veinte hasta nuestros días.

Una mirada cuantitativa es esclarecedora: en las décadas del 60 y 70 la proporción de personas nacidas en Paraguay que se encontraban residiendo en el exterior representaba entre el $10 \%$ y el $14 \%$ de la población total, siendo la cifra más alta de América Latina. En nuestros días se estima que alrededor del 7\% de la población total de Paraguay vive en el extranjero, un porcentaje solo superado por El Salvador (Texidó, 2003). ${ }^{1}$ En términos netos, las estimaciones señalan que desde 2001 hasta 2007 la emigración ascendió a 280.000 personas, lo cual abarca a una décima parte de la población económicamente activa de Paraguay (PNUD, 2009).

Según datos de la Encuesta Permanente de Hogares de Paraguay (EPH-P), el número estimado de migrantes en el quinquenio 2005-2010 fue de 170.000 personas, las cuales proceden en mayor medida de áreas rurales. ${ }^{2}$ El departamento Central es el que contribuyó en mayor medida al flujo total de emigrantes, aportando cerca del $25 \%$ del total, seguido luego por el departamento de Itapúa con 17\% aproximadamente, Caaguazú y Alto Paraná con alrededor del 10\% cada uno.

1 Halpern (2009) señala que algunas estimaciones realizadas por investigadores con una extensa trayectoria en la materia han calculado que el porcentaje de población paraguaya que vive fuera de país se ubicaría entre el 10 y el $15 \%$.

2 A pesar de la magnitud y el dinamismo del fenómeno migratorio, saber quiénes se fueron, al menos en términos sociodemográficos, no es tarea sencilla. En el caso de nuestro análisis, una debilidad importante radica en que en muchos relevamientos estadísticos las preguntas migratorias se aplican a una muestra, por lo que el número de personas pertenecientes al colectivo paraguayo suele ser reducido o no permite el análisis desagregado de un conjunto amplio de indicadores. Frente a estas limitaciones, y con el objetivo de aproximar la caracterización de las y los migrantes y sus lugares de origen, se trabaja en primer lugar con datos de la Encuesta Permanente de Hogares del Paraguay (EPH-PY). Específicamente, se ha procesado la base de datos correspondiente al último trimestre del año 2010. Deben tenerse en cuenta para la interpretación de la información las limitaciones que presenta el estudio de la emigración a través de fuentes de datos del país emisor, como lo es en nuestro caso la EPH relevada en Paraguay: a) no permite la captación de los casos en que haya emigrado todo el hogar con todos sus miembros; b) no permite la captación de aquellos migrantes cuyos miembros en el hogar en el país de origen hayan fallecido. 
Entre las características sociodemográficas de los emigrantes de nuestros días se destaca la preponderancia de mujeres (54\%), tanto de las áreas rurales como las urbanas, lo cual confirma el proceso de feminización de la migración que se ha registrado en las últimas décadas. ${ }^{3}$

CUADRO 1: MIGRANTES POR EDAD AL PARTIR, SEGÚN SEXO.

PARAGUAY, 2005-2010

\begin{tabular}{|l|c|c|c|}
\hline \multirow{2}{*}{ Total } & \multicolumn{3}{|c|}{$\mathbf{2 0 0 5 - 2 0 1 0}$} \\
\cline { 2 - 4 } & Total & Varones & Mujeres \\
\cline { 2 - 4 } & $\mathbf{1 7 5 . 7 3 1}$ & $\mathbf{8 0 . 8 5 2}$ & $\mathbf{9 4 . 8 7 9}$ \\
\hline Edad al emigrar & & & 4,5 \\
\hline $0-14$ & 3,9 & 3,3 & 21,5 \\
\hline $15-19$ & 21,6 & 21,8 & 30,7 \\
\hline $20-24$ & 35,3 & 40,8 & 15,8 \\
\hline $25-29$ & 16,2 & 16,7 & 11,0 \\
\hline $30-34$ & 9,6 & 8,0 & 5,7 \\
\hline $35-39$ & 4,5 & 3,0 & 10,9 \\
\hline 40 y más & 8,8 & 6,4 & \\
\hline
\end{tabular}

Fuente: Elaboración propia en base a datos de la EPH-PY.

En los departamentos más populosos del país, tales como el departamento Central y el del Alto Paraná, el peso de las mujeres en la emigración se incrementa a cerca del $60 \%$. Un rasgo fundamental relativo a la composición demográfica de los migrantes de ambos sexos radica en que se trata de una emigración eminentemente joven. Casi tres de cada cuatro migrantes tenían entre quince y veintinueve años de edad

3 El proceso de «feminización» de las migraciones trasnacionales ha sido ampliamente estudiado durante los últimos veinte años. Los estudios feministas han dado cuenta de este proceso, analizando cómo la mujer se ha convertido en protagonista de la migración internacional. En efecto, mientras hace unas décadas las razones de movilidad femenina estaban asociadas principalmente a motivaciones de «reunificación familiar», en nuestros días la migración se enfoca en búsqueda de trabajo e ingresos propios a la mujer. Los conceptos de cadenas globales de cuidado y familias trasnacionales son centrales dentro de este corpus. Para mayor detalle: Pérez Orozco, Amaia (2009): «Miradas globales a la organización de los cuidados en tiempos de crisis I: ¿qué está ocurriendo?» Santo Domingo. UN-INSTRAW. Serie Género, Migración y Desarro1lo. Documento de Trabajo $\mathrm{N}^{\circ} 5$. 
al momento de partir (cuadro 1). Y, dentro de este grupo, los/las jóvenes entre veinte y veinticuatro años fueron quienes migraron en mayor medida, superando el 35\% del total. En este sentido, cabe destacar además que en las áreas rurales el peso de la juventud en la emigración es aún más significativo y alcanza al $90 \%$.

Los datos confirman las tendencias observadas en las investigaciones del campo sobre la preponderancia de la población joven en el corredor migratorio (Sanchís y Rodríguez Enríquez, 2011). La motivación de buena parte de la juventud paraguaya por buscar alternativas como la migración puede comprenderse a la luz de aspectos estructurales que limitan sus horizontes. En efecto, uno los factores determinantes en las transiciones juveniles de muchos jóvenes es la falta de oportunidades laborales. En este contexto, uno de los rasgos fundamentales que caracterizan a la juventud paraguaya es su temprana vinculación con el mercado laboral. Más del $45 \%$ de los varones y algo menos del $30 \%$ de las mujeres que han trabajado alguna vez, obtuvieron su primer trabajo remunerado antes de los 15 años, lo cual integra a los jóvenes de ambos sexos - de manera temprana - a una fuerza de trabajo «disponible», a ser interpelada por las oportunidades de los mercados de trabajo de las economías en etapa de crecimiento.

Otra característica relativa al corredor migratorio paraguayoargentino está relacionada a que el patrón migratorio corresponde con la partida de las personas con anterioridad a la formación de un núcleo familiar propio (Sanchís y Rodríguez Enríquez, 2011). Al respecto, Cerrutti (2009b) ha hallado una amplia proporción de paraguayas que encaraban la movilidad de manera independiente, lo cual, se sostiene, está relacionado con el posicionamiento femenino al interior de la familia agrícola. Según la autora, el lugar de la mujer en la provisión de ingresos extrafamiliares conforma y delimita su participación en actividades del mercado informal, representando un antecedente central de los procesos migratorios (Cerrutti, 2009a), en donde la migración femenina proveniente de hogares campesinos adquiere una importancia fundamental.

La información detallada hasta aquí posiciona a los/las jóvenes en el centro de la escena, en tanto protagonistas fundamentales del fenómeno migratorio: una movilidad juvenil encarada mayoritariamente de forma individual y que se da en un momento de emancipación o búsqueda de un lugar propio. Justamente, la juventud corresponde con un período vital de búsqueda y autonomización familiar, en donde los recursos disponibles son utilizados y combinados según el posiciona- 
miento social y de género. En las entrevistas se ha propuesto avanzar en el conocimiento de cómo estas oportunidades se convierten en trayectorias de inserción en el país de destino.

\section{LAS CONDICIONES DE VIDA}

DE LA JUVENTUD PARAGUAYA EN ARGENTINA

La inserción social y económica de los/las jóvenes inmigrantes asume diversos aspectos y su medición precisa resulta de extrema dificultad. A pesar de ello, pueden considerarse ciertos datos que nos orienten en cuanto a la forma y el grado de inserción en el contexto receptor. Las dimensiones educativa y laboral son, sin dudas, algunas de las más importantes.

CUADRO 2: INDICADORES EDUCATIVOS Y ACTIVIDADES DE JÓVENES ENTRE 18 Y 29 AÑOS MIGRANTES PARAGUAYOS. GRAN BUENOS AIRES, 2005-2010*

\begin{tabular}{|l|c|c|c|}
\hline Indicadores & Total & Varón & Mujer \\
\hline Asistencia escolar & & & \\
\hline Asiste & $8,6 \%$ & $5,9 \%$ & $10,4 \%$ \\
\hline Finalización de la secundaria & & & \\
\hline Secundaria completa & $43,4 \%$ & $40,8 \%$ & $36,7 \%$ \\
\hline Actividades & & & \\
\hline Estudia & $3,7 \%$ & $2,3 \%$ & $4,5 \%$ \\
\hline Estudia y busca trabajo & $1,0 \%$ & $1,0 \%$ & $1,1 \%$ \\
\hline Estudia y trabaja & $3,9 \%$ & $2,6 \%$ & $4,8 \%$ \\
\hline Es inactivo y no estudia & $18,1 \%$ & $3,6 \%$ & $27,7 \%$ \\
\hline Es desocupado y no estudia & $9,5 \%$ & $9,9 \%$ & $9,3 \%$ \\
\hline Trabaja & $63,7 \%$ & $80,6 \%$ & $52,6 \%$ \\
\hline
\end{tabular}

Fuente: Elaboración propia en base a la EPH-A, INDEC.

* A fin de contar con una mayor cantidad de casos que permita el análisis del grupo poblacional específico de interés, en el procesamiento de los datos se consolidaron las bases de la EPH-A desde el año 2005 hasta el 2010. La información debe ser utilizada para la comparación entre subgrupos dentro de la misma base y no es representativa de la población total.

Según información de la Encuesta Permanente de Hogares de la República de Paraguay (en adelante EPH-PY), la educación no resulta ser la razón principal que impulsa la movilidad. De forma consistente, los datos de la Encuesta Permanente de Hogares de Argentina (EPH-A) dan 
cuenta de que la juventud inmigrante paraguaya mayor de 18 años no continúa estudios en la Argentina como lo demuestra el hecho de que menos del 10\% presenta asistencia a una institución escolar (cuadro 2). En cualquiera de los grupos de edad o sexo que se considere, los y las jóvenes desarrollan su vida por fuera del sistema educativo local. Respecto a los activos o logros educativos con que han emigrado, puede afirmarse que una gran parte no ha alcanzado a finalizar el nivel medio. Específicamente, más del 55\% no concluyó el nivel medio y, como se ha visto, es ínfimo el porcentaje que continúa estudiando. Entre las mujeres, el porcentaje que representa quienes terminaron el secundario es aún menor que el de los varones con un $63 \%$ y 59\%, respectivamente.

Analizando sus actividades, se observa que la amplia mayoría de la juventud migrante -8 de cada 10 - se encuentra trabajando o en la búsqueda de empleo y lo cual se confirma desde sus tasas de actividad. Para los jóvenes varones el estar económicamente activo es prácticamente una realidad; para las mujeres, a pesar de ser algo menor que en aquéllos, la actividad también es muy elevada, ubicándose entre el 80 y $85 \%$.

Con referencia a las condiciones de trabajo, los datos disponibles confirman que el incremento del trabajo registrado de los últimos años, ocurrido en un contexto de crecimiento económico y una importante reducción del desempleo general, no alcanzó a la juventud paraguaya inmigrante. Su inserción sigue mercada por una fuerte precariedad laboral, encontrándose en esta situación casi nueve de cada diez jóvenes. La edad y el sexo influyen en cierta medida en el grado de precariedad, acentuándose entre las mujeres y reduciéndose a medida que aumenta la edad de los jóvenes.

CUADRO 3: INDICADORES LABORALES DE JÓVENES ENTRE 18 Y 29 AÑOS MIGRANTES PARAGUAYOS. GRAN BUENOS AIRES 2005-2010

\begin{tabular}{|l|c|c|c|}
\hline \multirow{2}{*}{ Tasas } & \multirow{2}{*}{ Total } & \multicolumn{2}{|c|}{ Sexo } \\
\cline { 3 - 4 } & & Varón & Mujer \\
\hline Tasa de actividad & 78,2 & 94,1 & 67,8 \\
\hline Tasa de empleo & 67,7 & 83,2 & 57,5 \\
\hline Tasa de desocupación & 13,5 & 11,5 & 15,3 \\
\hline Aportes jubilatorios & & & \\
\hline Sin aportes & $79,1 \%$ & $73,9 \%$ & $83,9 \%$ \\
\hline Tipo de contrato & & & \\
\hline Temporario & $78,1 \%$ & $75,1 \%$ & $85,2 \%$ \\
\hline
\end{tabular}

Fuente: Elaboración propia en base de la EPH-A, INDEC. 
En el análisis de la inserción laboral de los jóvenes migrantes resulta imprescindible abordar el estudio sobre las ramas de actividad en que se desempeñan. Al respecto, se ha señalado que la migración se constituye en un esquema de dificultad en el lugar de origen y de atractivo del espacio al lugar al que se llega. Un claro ejemplo de estas circunstancias se constituye a partir de las mencionadas cadenas globales de cuidados. El concepto de cadenas de cuidado sostiene que en los lugares de destino la dinámica de la incorporación de las mujeres al mercado laboral crea al mismo tiempo una crisis en la atención de personas con requerimientos especiales que termina dando forma al segmento en el mercado laboral donde las migrantes se desempeñan. Así, la externalización y mercantilización de los cuidados se resuelven de forma privada en base a la contratación de mano de obra migrante (Sanchís y Rodríguez Enríquez, 2011). De forma tal que las ocupaciones del servicio doméstico y de cuidados siguen representando las opciones más representativas de las jóvenes paraguayas, como se verá luego en el apartado que detalla las entrevistas.

En esta misma dirección, las características del crecimiento económico de los últimos años implicaron la revitalización del segmento informal de la construcción como principal demandante de jóvenes paraguayos. Los datos de referencia dan cuenta de que entre prácticamente siete de cada diez migrantes se desempeñan en esta rama de actividad, la cual se establece como la contracara de la modificación de la tendencia a la feminización del corredor migratorio.

Los datos analizados permiten afirmar que la migración parece acelerar definitivamente el salto hacia la adultez en tanto que determina para casi todos los jóvenes la finalización del período formativo y la participación en la actividad económica. Asimismo, en el proceso los jóvenes suelen dejar el hogar de origen, lo cual marca el comienzo de la transición hacia la conformación del propio. En muchos casos, pasan a vivir solos alquilando una pieza, en otros se reside un tiempo con parientes mientras se alcanza cierta estabilidad laboral y se intenta conseguir un lugar propio, ya sea compartido o no. En cualquier caso, la migración implica la mayoría de las veces el alejamiento del hogar de origen y conlleva responsabilidades económicas y decisiones en torno a un nuevo hogar que marca avances en el proceso de autonomización propio del período juvenil. 
GRÁFICO 1: DISTINTOS MOMENTOS DEL PROCESO MIGRATORIO

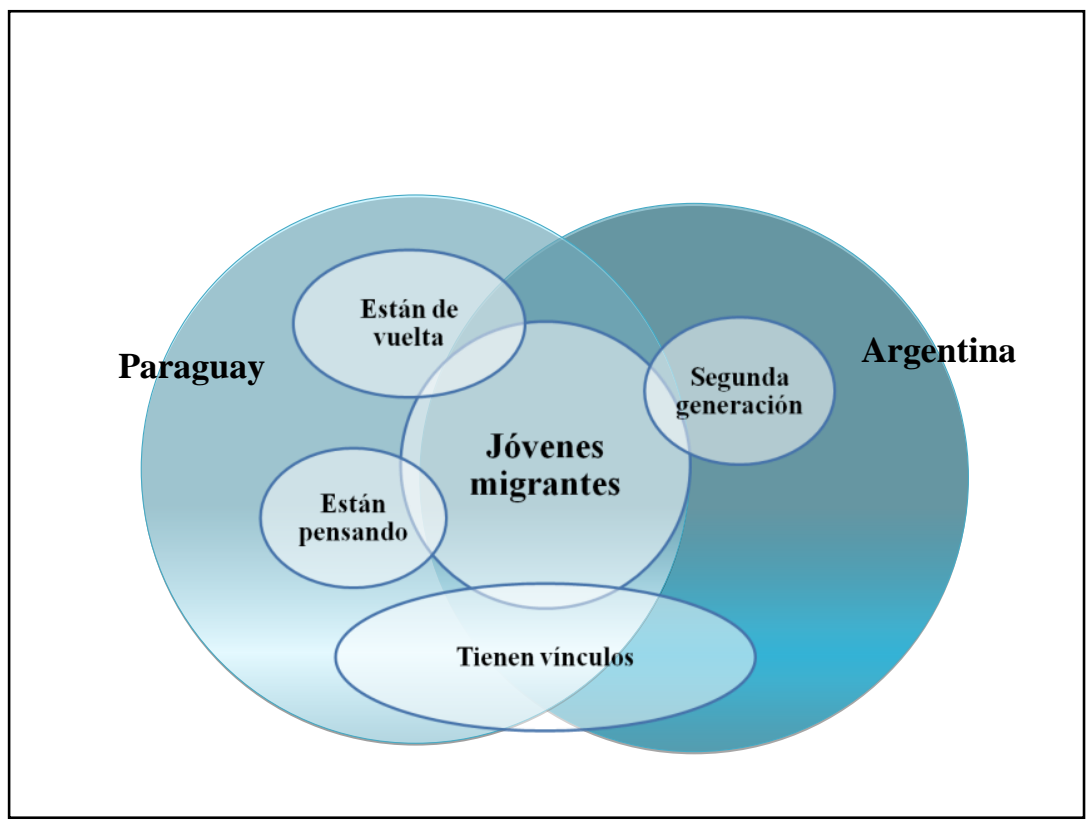

\section{RELATOS DEL PROCESO MIGRATORIO Y CONDICIONES DE VIDA}

Los relatos de los/as jóvenes ocupan un lugar central en el estudio del proceso migratorio. En este sentido, durante la investigación se trabajó con entrevistas a distintos actores vinculados al corredor paraguayoargentino. Las entrevistas fueron realizadas en distintas ciudades de ambos países. Se distinguieron cinco grupos según etapas del proceso migratorio. De esta forma, del lado paraguayo se entrevistó a: i) jóvenes que habían migrado y están de vuelta en Paraguay; ii) jóvenes que están pensando en migrar; iii) jóvenes que tienen vínculos directos con la migración a través de familiares. Y del lado argentino se entrevistó a: i) jóvenes migrantes y ii) jóvenes hijos de migrantes. En todos los casos, el rango de edad estuvo delimitado por los 18 y 29 años. En el gráfico 1 se representan los distintos grupos o tipos migratorios con los que se trabajó durante la investigación.

El trabajo de campo comprendió un total de cincuenta entrevistas. De las cuales, treinta entrevistas se realizaron en el Gran Buenos Aires entre jóvenes migrantes de primera y «segunda» generación. Mientras que otras veinte entrevistas se realizaron en distintas ciudades de $\mathrm{Pa}$ - 
raguay. Dadas las limitaciones de extensión de la ponencia, a continuación se detallan los resultados de veinte entrevistas realizadas entre quienes emprendieron la migración durante su período juvenil.

\section{a) Proceso migratorio y cambios en las trayectorias de vida}

A partir de la lectura de los relatos, resulta muy dificultoso hacer referencia a un «proyecto» migratorio como tal. En las entrevistas realizadas se encontró una cuota importante de indeterminación en la proyección del viaje que realiza el o la joven o su familia a la Argentina. Es decir, frecuentemente las afirmaciones expresaban frases con cierta ambigüedad, tales como: «vine por unos meses», «vine a conocer», «vine a la Argentina y si lograba progresar me quedaba», «quería ver si me hallaba», «me insistieron para que viniera», «acá estaba mi familia, pero extraño», etcétera. Algunos entrevistados vinieron a la Argentina unos meses o algunos años, regresaron a Paraguay para terminar sus estudios o por otros motivos y nuevamente migraron a Buenos Aires, mostrando que en muchos casos no se trata de un proceso unilineal o definitivo. Mientras conversábamos con ellos, algunos manifestaban que extrañaban a su país y que esperaban volver, pero lo harían solo si se cumplen ciertas condiciones; es decir, por ejemplo, si logran tener suficientes ahorros para abrir un comercio en su país o poder comprar una casa y no pasar penurias económicas. Así encontramos que muchos están atentos a cómo se encuentra la situación económica en su país, buscando reportes en los llamados que frecuentemente realizan a sus allegados en sus localidades de origen. Sólo algunos hicieron referencia a que ya no se hallarían (cómodos) en Paraguay; es decir, sostenían que se acostumbraron a la «forma de vida argentina» $y$, por lo tanto, se trataría ya de un proceso irreversible.

\section{b) Todos los entrevistados contaban con alguna red de apoyo en Argentina antes de iniciar el proceso migratorio}

También fue recurrente el relato de lo duro que fue despedirse en su localidad de origen, con sus parientes más cercanos presentes, los que lo o la acompañaron hasta la terminal de ómnibus, medio de transporte utilizado por la inmensa mayoría de los jóvenes. Algunas entrevistadas también nos relataron los miedos que sentían durante el traslado, si se encontraban solas, hacia un lugar desconocido y anticipado por sus referencias como diferente a su país. No obstante, una porción 
importante de los entrevistados ya había experimentado previamente traslados en su lugar de residencia en Paraguay, ya sea cuando eran niños, cuando se encontraban en la adolescencia o comenzando su juventud. Estas mudanzas se debían a reorganización familiar, por motivos de estudios, trabajo o salud. En muchos casos se trató de migración interna rural-urbana.

\section{c) Las redes de parientes y conocidos es el elemento central para canalizar el proceso migratorio}

Un hallazgo de la investigación indica que las redes de parientes y conocidos es el elemento central para canalizar el proceso migratorio, coincidente con lo que plantean Cerrutti y Parrado (2006) e inclusive para elegir el lugar donde comenzar una nueva etapa. Se observó que, por lo general, se trata de «redes femeninas» y «redes masculinas» de manera diferenciada, aunque existen excepciones. En muchas oportunidades se produce incluso una situación de «invitación»: que algún pariente que vive en la Argentina avisa de una oportunidad laboral, en algunos casos ya sabiendo el interés del joven que se encuentra en Paraguay. Por ejemplo, alguna empleada doméstica que está por dejar su trabajo le avisa a su empleadora que tiene una hermana, una prima o una amiga que puede ocupar su puesto. Se produce entonces una cadena de relaciones de confianza entre empleada y empleadora que resulta proveedora de recursos humanos para su reemplazo, oficiando de «recomendadora» y «garantía». En los relatos fue frecuente que quienes vivían en la Argentina les comunicaban que era fácil conseguir trabajo o que pagaban mejor que en Paraguay, que era posible estudiar o atender la salud sin tener que desembolsar dinero. Algunos, ya habían estado en el país de vacaciones, en cambio otros comenzaron el trayecto migratorio sin conocer Buenos Aires previamente. Todos tenían referencias del país, e incluso varios entrevistados detallaban cómo sus parientes en Argentina les explicaban cómo funcionaban las cosas (transporte, trámites, relaciones laborales, vivienda, etcétera.), como lo hace Ignacio (20), ${ }^{4}$ que nos explicaba cómo funcionaban las redes y los motivos de sus compatriotas:

4 Los nombres de los entrevistados fueron cambiados a fin de preservar su identidad. 
mis tíos habían venido por un vecino, que este vecino y su hermano habían venido acá para trabajar y como le consiguió trabajo a mis tíos y bueno... vinieron y luego estos tíos trajeron a sus hermanos menores, ¿no? Dos tíos más grandes que tengo yo... y supongo que habrá sido buscar un mejor trabajo porqué... no sé si en ese momento el peso ya valía más que el guaraní, no sé, pero por ahí pudo haber sido eso, no sé. El factor económico principalmente.

Otro entrevistado, Guillermo, sintetizaba su decisión de la siguiente forma:

yo decidí esto de sorpresa, porque así como te dije, como mi situación no era buena [se había quedado sin empleo en Ciudad del Este] y como tenía parientes acá, ellos se fueron y me dijeron si quería venir, que ellos me verían un trabajo en donde sea, en algún lugar te vamos a conseguir, me dijeron..., yo soy trabajador, no quiero estar al pedo, no quiero andar de haragán, y entonces, tomé la mochila en la espalda y vine, así mismo nomás como estaba.

Sus ocho hermanos quedaron allí (otros dos murieron), pero fueron llegando después y él reflexiona sobre su condición de pobreza y cómo desde muy temprana edad cada uno debía procurarse ingresos:

mi papá estaba separado de mamá, que tiene 82,85 , y papá siempre de aquí para allá, anduvo, y la que nos crió fue mamá, o sea es una familia bastante pobre, entonces nosotros, creo que la consecuencia de todo eso es que termine viniendo acá. Porque cada uno nos teníamos que arreglar, rebuscar cada uno con su necesidades.

De esta forma, se vive naturalizadamente el destino migratorio, como parte de la vida de los miembros de una familia pobre.

\section{d) Los jóvenes perciben que el proceso migratorio les daría una mayor libertad de sus padres}

Por otra parte, varios entrevistados también consideraban que el proceso migratorio los llevaría a un lugar de mayor libertad en relación a sus padres. En un caso, un muchacho gay, sentía que el proceso migratorio lo liberaba de la mirada de su ciudad natal, plagada de señalamientos y discriminación social. Con lo cual para el joven migrante existe una tensión permanente entre la autonomía y el mantenimiento de las redes familiares a la distancia o en proceso de reunificación 
familiar. Pero como sosteníamos para lograr la autonomía, en relación a autosustentarse e inclusive obtener una vivienda propia (en alquiler o en propiedad) las redes de connacionales en el lugar de destino son el recurso indispensable y reiterado en los relatos. Esta autonomía implica aprendizajes, como lo indicó un entrevistado: «aprendí a cuidarme solo, aprendí a cuidarme solo, aprendí a desenvolverme solo, aprendí a usar colectivo, a usar... que sé yo, ¡el tren!, nunca, nunca en mi vida me subí en un tren, nunca en mi vida me subí en un subte».

\section{e) Motivaciones de la juventud para migrar a la Argentina}

En muchas oportunidades los jóvenes indagados manifestaron la necesidad de consumo como un motivo del empleo temprano y la idea que circula en diferentes localidades de Paraguay es que en Buenos Aires accediendo al trabajo se accede a mayor consumo. Ana nos afirmaba: «como todo joven, necesitaba..., uno quiere todo y mi familia no me podía dar lo que yo necesitaba, yo vivía con mi mamá, mi padrastro y es como que mi padrastro se quedó sin laburo y no me podía ayudar económicamente, termine mi bachiller y me vine para acá». En este caso además, como en otros, se planificó la migración con la finalización de la secundaria, aunque no siempre sucede así. Es decir, no solamente la necesidad imperiosa de obtener trabajo era el motivo, sino también la atracción del modo de vida urbano, tal como lo relata Iván (20), cuando se le preguntó sobre la imagen previa de Buenos Aires:

Me acuerdo que era toda la ciudad moderna, con cosas raras vamos decir, me acuerdo que mi mamá se iba y nos contaba del semáforo, súper raro para nosotros... entonces tenía la idea de uou, una ciudad grande, con muchos edificios altos me imaginaba, mucha gente, autos, eso... esa imagen me atraía curiosamente, sí me atraía, pero supongo porque vi en la tele a New York, también por es (el subrayado es nuestro).

\section{f) Los jóvenes consideran que migran hombres y mujeres por igual}

Los entrevistados consideraban que migran de manera equivalente hombres y mujeres, aunque algunos hacen referencia que para las mujeres hay más trabajo y más rápido. No obstante Wainer, Lago y Gabea (2009) afirman en que se mantiene el estereotipo de que la mujer trabaja como empleada doméstica y el hombre en la construcción y los datos empíricos lo refuerzan. No obstante aparecen otras ocupaciones entre los jóvenes migrantes, como empleados en comer- 
cios o «en costura». En cuanto al primero, se trata sobre todo de ocupación femenina y el segundo más masculina. Los entrevistados hacen referencia que podrían obtener empleos más calificados, debido a que su baja escolaridad lo impide.

Migración y juventud están claramente asociadas, en particular antes de conformar una pareja estable. Por esta razón, al momento de emprender ese camino, la proporción de mujeres solteras llegaría a ser la mitad (Cerrutti y Parrado, 2006), muchas de ellas lo hacen entre 16 y 18 años. Empleo y escolarización también evidencian un relación que se repite en el caso de los migrantes, lo que conocen de esta situación y en algunos casos, si las condiciones se dan, buscan completar sus estudios secundarios para aspirar a trabajos no manuales.

\section{g) Percepción de los jóvenes respecto a lo que Argentina les ofrece}

La juventud paraguaya migrante coincide en que Argentina ofrece más oportunidades laborales, así como acceso a una calidad mejor y sin pagar en educación y salud. En las conversaciones con los entrevistados se afirmaba que los trabajos son mejor pagos en Argentina que en Paraguay, y ese es motivo de que parientes o amigos «inviten» a venir a trabajar aquí, por una temporada o quizás a quedarse. Inclusive, se relata que los argentinos pagan más que los paraguayos cuando son empleadores en Argentina.

Fue un dato reiterado en las charlas que en Argentina para acceder a la educación primaria no hace falta tener documento argentino, no obstante los jóvenes interrogados hacían referencia a que en algunos casos sufrieron la falta de una serie de documentos que acreditaran sus estudios en Paraguay, y en entonces en algunas ocasiones, los entrevistados no pudieron cumplimentarlos o traerlos, lo que frenó sus expectativas de continuidad de carrera educativa. Muchos se encontraban con expectativas de lograr acreditar sus estudios secundarios y poder en algún momento iniciar estudios superiores en Buenos Aires, en este último caso, era mayor para las mujeres que para los hombres. De acuerdo a los relatos comparativos entre padres y jóvenes migrantes, se observa una mayor escolaridad (aunque algunos datos son imprecisos), pareciendo predominar padres con primaria incompleta y en los jóvenes migrantes la secundaria incompleta (en menor medida completa).

5 De acuerdo a Wainer, Lago y Gabea (2009) en la Ciudad de Buenos Aires la población paraguaya presenta en su mayoría (78\%) un nivel de instrucción menor a secundario incompleto. Solo un 15,3\% alcanza el 


\section{h) Los jóvenes migrantes perciben la educación como un vector de ascenso social}

La educación es considerada como un vector de ascenso social. No obstante, en los relatos de los entrevistados apareció recurrentemente que su primer empleo comenzó alrededor de los catorce o quince años. En algunos casos inclusive antes, alrededor de los 10 años, pero por lo general en condición de «ayuda» al padre o madre, diferenciándose tempranamente el trabajo masculino del femenino. Por ejemplo, Ricardo (Ybicui, 25) explicaba: «En Paraguay... no te puedo decir de mi primer trabajo, ahí yo creo que empezás a trabajar desde el momento que sabes agarrar un machete, un hacha, una motosierra, sí, no te puedo decir...» (él trabajaba con el papá). Las mujeres lo hicieron como empleadas domésticas o de comercio, en este último caso ayudando a los padres en sus emprendimientos económicos. En otras oportunidades, comenzaron las labores auxiliando o a lavar ropa «para afuera», o cuidando a sus hermanos menores o en tareas vinculadas al campo, como ordeñar, carpir o cosechar. En el caso de los hombres, sus primeras tareas fueron como ayudantes en la construcción, transporte o en tareas agrícolas como carpir, cosechar, manejar la motosierra, cargar cosas, etcétera.

Por lo general, tanto para hombres como para mujeres este inicio laboral se asoció a tener que dejar de estudiar, ya que a pesar de intentarlo no pudieron mantener el ritmo de la escolaridad. En el relato de varios entrevistados se hizo referencia a que en Argentina se intentó retomar los estudios, en muchos casos esto fracasó por falta de documentación, de tiempo o fuerza para continuarlos. En aquellos que ya tenían el secundario completo o incompleto, en muchos casos se optó por realizar cursos con salida laboral, tales como computación, manicuría o electricidad. Solo en pocas ocasiones se optó por carreras universitarias. Una de nuestras entrevistadas, perteneciente a una familia de clase media con recursos en Paraguay, decidió el destino de Buenos Aires para continuar con su carrera de licenciatura en relaciones internacionales, ya que la calidad educativa sería mejor que la su país, según las averiguaciones que realizó antes de tomar la decisión. Esta es una situación excepcional, más bien, aquellos que tuvieron un buen

secundario completo (siendo ligeramente mayor en el caso de las mujeres). Muy pocos migrantes muestran niveles de estudio terciarios o universitarios (completos e incompletos). 
desempeño en su secundario (en su país o en Argentina) exponen su expectativa de estudio universitario más allá de que pudieran concretarlo o no, sin que se viera concretar.

Como sostiene García Borrego (S/F), lo que diferencia a los/las jóvenes migrantes de sus pares en la sociedad en la que se insertan es que la transición de la escuela al trabajo (en el nivel que se haya podido alcanzar) o de la adolescencia a la adultez es directa. Es decir, la postergación de la independencia económica y en muchos casos habitacional que se observa en aquellos que permanecen en su lugar de origen es difícil hallarlo en los jóvenes migrantes. En las entrevistas es constante la comparación entre las condiciones laborales paraguayas y argentinas y las experiencias de las jornadas y relaciones en el trabajo.

\section{i) Los jóvenes migrantes y los tipos de trabajos que desempeñan}

La presente investigación confirma la imagen de que las jóvenes migrantes paraguayas trabajan en empleos vinculados al servicio doméstico (mucamas, niñeras, cocineras, cuidadora de mayores) o limpieza de oficinas, en algunos casos como primer empleo con «cama adentro» o cuando son mujeres separadas que viajan solas. En los relatos surge que la opción «cama adentro» no genera una situación agradable para las migrantes (agravado por estar recién llegadas al nuevo país), quienes apenas pueden cambian a la condición «con retiro» No obstante, el trabajo en el sector comercio emerge como otra ocupación relevante. Para ello, las entrevistadas hacían referencia a un «aprendizaje» de saber cómo relacionarse con las personas y manejar bien el idioma español. Otras realizan emprendimientos, como pequeños comercios. En algunos casos, las jóvenes madres se dedican exclusivamente al cuidado de sus hijos.

En el caso de los hombres, el trabajo que predomina es el de albañilería y otros oficios vinculados a la construcción. ${ }^{6}$ En un contexto de crecimiento económico en Argentina (donde se presenta un boom constructivo y de obra pública) y dado que el trabajo vinculado a la

6 Tomando como dato la ciudad de Buenos Aires, en el 2001 (teniendo presente la particularidad del año, en cuanto al alcance de la crisis económica) el 78,4\% de los paraguayos ocupados trabaja como obrero/empleado en el sector privado, un $13,4 \%$ como cuenta propia y el resto como empleado público, trabajador familiar y otros (Wainer, Lago, Gabea, 2009). 
industria de la construcción recluta particularmente jóvenes, los paraguayos son mano de obra preponderante, sobre todo en aquellos emprendimientos de menor escala o formalidad. Algunos otros trabajan como operarios en fábricas, empleados de comercio o por cuenta propia, como por ejemplo, talleres mecánicos.

\section{j) Los jóvenes migrantes perciben que los argentinos} tienen una imagen positiva de ellos en el ámbito laboral

Los entrevistados coincidieron en señalar que los argentinos tienen una imagen positiva de los paraguayos en el ámbito laboral (aunque no exenta de discriminación o mejor de subordinación). En esta dirección, Ana explicaba con relación al trabajo femenino:

porque los argentinos quieren mucho a los paraguayos para que trabajen en la casa, porque somos muy trabajadoras, muy honestas, muy responsables, bueno no todas pero la mayoría sí, por eso nos quieren más a los paraguayos.

Se utilizó la palabra «guapa» para hacer referencia a la capacidad de trabajo de las jóvenes paraguayas, que encaran con responsabilidad y compromiso las tareas que asumen. En el caso de los hombres Gustavo (Villarrica, 29) afirmaba «el paraguayo se preocupa por su trabajo... deja todo por el trabajo... se las arregla... así va avanzando». Se destacaba la posibilidad de defender sus derechos laborales que ofrece la Argentina.

\section{CONDICIONES DE VIDA}

\section{a) Los jóvenes migrantes destacan diferencia de calidad de vida entre Paraguay y Argentina}

En todos los relatos de los entrevistados se remarcó la diferencia de la calidad de vida entre Paraguay y Argentina y este factor como un motivo para quedarse, aun cuando se quiera volver. No obstante, como afirma García Borrego (S/F), los jóvenes migrantes tienen que recorrer los mismos caminos que el resto de personas de su edad, pero tienen que hacerlo en peores condiciones que ellos, porque a los dos itinerarios habituales (de la escuela al trabajo y de la adolescencia a la adultez) se añade un tercero, el que va de la sociedad de su país de origen a la receptora. 
Migración y vivienda tienen una estrecha relación inversa. No solo porque el que posee una vivienda es reacio a migrar, sino porque el que migra en muchos casos envía remesas para la construcción de su propia casa o realiza ahorros para tal efecto para cuando vuelva, en el período pos-productivo. Al igual que sucede en muchos países latinoamericanos, muchos utilizan los ahorros producidos durante la migración para construir su vivienda en Paraguay.

Para los migrantes internos y regionales existen fuertes restricciones para acceder a un departamento o casa en alquiler (Cravino, 2006) en el mercado inmobiliario formal de la Ciudad de Buenos Aires, ya que se les exige una o dos garantías (alguien que avale y que posea una propiedad dentro del distrito capitalino), sumado a la presentación de documentos argentinos y de trabajo registrado. Por esta razón, gran parte de los recién llegados a Buenos Aires en busca de trabajo y oportunidades se ve compelido a vivir en un asentamiento informal («villa»o «asentamiento») y las redes sociales son centrales para acceder a este tipo de viviendas (Cravino, 2006). La mayoría de los migrantes regionales se asienta en la Capital Federal por la presencia de parientes o amigos y porque allí existen más fuentes de trabajo. Al mismo tiempo, allí se puede consumir comidas típicas, escuchar música de las distintas nacionalidades, participar de fiestas, jugar al voley o comprar productos paraguayos.

Algunas restricciones semejantes se observan en el Gran Buenos Aires. El alquiler de un cuarto (con baño compartido) en inquilinatos y de un departamento o casa es la forma habitual de entrada al barrio en los distintos asentamientos informales del Área Metropolitana de Buenos Aires (Cravino, 2006, 2008a). Por su parte, aquellos que llevan varios años viviendo en las villas y no se encuentran en condición de inquilinos suelen construir cuartos (por lo general en altura) para alquilar a sus compatriotas y así tener un ingreso adicional. De esta forma, así como la juventud migrante ingresa al mercado laboral con peores condiciones, lo mismo sucede en cuanto al lugar que habita en la ciudad.

Ignacio explicaba: «no es que uno se va y progresa fácil, sino que uno se va y sacrifica la vida y acá vemos que están viviendo en la villa así, también con mucha ausencia del estado». En la periferia del Gran Buenos Aires los precios de los lotes o las viviendas son muchos más económicos y algunos/as migrantes lograron constituirse en propietarios/as. En estos barrios se suele dar una fuerte sociabilidad y en los relatos encontramos varios casos donde los/as jóvenes encontraron a su pareja en el inquilinato que compartían. 


\section{b) Los jóvenes migrantes y la sociabilidad en el nuevo país}

Los jóvenes migrantes, en Argentina, mantienen la sociabilidad en base a la familia, a relaciones vecinales previas en Paraguay o a nacionalidad. Las mujeres, en particular, no salen mucho de su casa, y utilizan su tiempo libre para descansar, arreglar su vivienda o visitar parientes, solo algunas «pasean» o salen a bailar. Los hombres expresan, en mayor proporción, salir a bailar y «tomar» (alguna bebida) entre amigos. Algunos juegan al voley o fútbol. Algunas mujeres también juegan al voley. En buena medida, se consumen productos traídos de Paraguay y hay vendedores especializados en tales productos (yerba, ropa, electrodomésticos, etcétera). Muchos de los jóvenes entrevistados escuchan radios de la colectividad paraguaya. Algunos incorporaron música de otros países, como la cumbia, en cambio, otros la rechazan. Un dato relevante que se desprende de las entrevistas es que no hay, en la mayoría de los casos, participación en organizaciones propias de la colectividad paraguaya. En algunos lugares quizás no las haya en la zona o se encuentran alejadas para la movilidad cotidiana.

En los relatos se hace referencia a que muchos jóvenes utilizan internet, en particular las redes sociales como facebook, para comunicarse con amigos, tanto de Argentina como de Paraguay. Prácticamente todos/as los/as entrevistados hicieron referencia a que tenían teléfono celular y que lo utilizaban para comunicarse, por lo general una vez por semana, cada dos semanas o en ocasiones con algunos allegados. La mayoría de sus parientes también contaba con telefonía móvil en Paraguay, solo algunos explicaron que llamaban a casa de vecinos porque sus familiares no tenían teléfono, estos eran casos de zonas rurales. Algunos jóvenes también utilizaban el computador para jugar. Algunos tienen computador propio y otros recurren a locutorios. El fútbol también es un factor de unidad entre la colectividad, y muchos se mantienen al día con la información y mantienen su identidad, como Darío (Caapucu, 23), que expresaba ser seguidor de Cerro Porteño pero al mismo tiempo había sumado su afición por River Plate. Muchos entrevistados afirmaban escuchar radios o canales paraguayos (por cable), así como programas producidos por compatriotas en Argentina (como por ejemplo Murucuyá). Como afirma García Borrego (S/F) estas personas no tienen únicamente proyectos migratorios, porque no son solo migrantes, son también jóvenes. 


\section{c) El idioma guaraní: ventajas y desventajas}

El idioma guaraní apareció muy tematizado en las entrevistas. En primer lugar como una gran dificultad para la inserción en Argentina. Para muchos fue la lengua materna, mientras que el español fue aprendido posteriormente y en particular en la escuela. Por otra parte, es un elemento de unidad entre la comunidad paraguaya y de identificación positiva con su lugar de origen, como una costumbre a conservar. En algunos casos hay referencias de que los argentinos (o personas de otras nacionalidades) sienten molestia por no poder entender qué hablan, en particular en ámbitos laborales, mientras que otros tienen inquietud de aprender algunas palabras en guaraní. Ricardo explicaba su adaptación al cambio de idioma, pero rescatando lo positivo como identidad que significa el guaraní:

Fue duro, también la idioma, la idioma, porque normalmente en el campo se hablan en guaraní, se habla en guaraní, vista la idioma también te cuesta poco, yo... a mí me costó un poco, porque, me ayudó mucho, me salí de mi casa irme a la ciudad, eso de ir y caminar 11 kilómetros, irme a la colonia donde la mayoría hablaba castellano, eso me ayudó un poco pero no mucho, y acá todo esto aprendí, a hablar, saber.. como te digo.. a hablar. Hablaba también castellano pero no con mucha fluidez, me faltaba fluidez. Y acá me tocó, me tocaba hablar...otra cosa es la timidez, no, no.. uno que viene del campo tiene mucha vergüenza, tiene mucha vergüenza de hablar, de equivocarse, yo eso lo entiendo, de un error se aprende otro. [...] es lindo juntarte con tu paisano y hablar en guaraní, saber que lo que hablas es tu idioma, pero no te ayuda en nada en el sentido de estar acá [...] allá es totalmente diferente, allá usan el guaraní y el castellano, usan todo mezclado, te hablan un poco en castellano y un par en guaraní, pero es lo que nos identifica a nosotros, es lo que nos identifica como paraguayos.

No obstante, la explicación de que la mixtura de dos idiomas es la culpable de que finalmente los paraguayos no sean comprendidos idiomáticamente por los argentinos fue repetida muchas veces. En relación al guaraní, la mayoría afirma hablarlo, pero no necesariamente escribirlo. Se sostiene que se utiliza para conversar con amigos o con compatriotas. En algunos casos se dan situaciones de complicidad en el trabajo (en particular en el rubro de la construcción) el compartir el idioma, pero que no siempre esto es bien visto por los empleadores o los mismos compañeros de trabajo. En algunas entrevistas se asocian dificultades con el español con timidez, en particular en las mujeres. 


\section{d) El uso del dinero y la comunicación con la familia de origen}

Las remesas, a pesar del cambio monetario peso-guaraní menos favorable, es una práctica recurrente en casi todos los entrevistados, tanto hombres como mujeres. Algunos autores indican el envío anual en 100 millones de dólares para fines de los años 90 (Halpern, 2009) y es considerada la segunda fuente de ingresos de Paraguay, después de la exportación de soja.

Muchos de los/as entrevistados/as enviaban remesas a sus padres, a sus hijos o algún otro pariente viviendo en su país de origen. Las remesas no parecen ser muy condicionadas en sus usos por quienes envían el dinero. En algunos casos los envíos dependen de pedidos puntuales desde Paraguay y, por lo tanto, cumplen el papel de cubrir emergencias, imponderables. Se suele enviar el dinero por medio de la empresa Western Union o alguna empresa de transporte terrestre. ${ }^{7}$ En el primer caso se obtiene descuento por ser cliente. La mayoría de los giros son mensuales, aunque es frecuente que algunos los realicen cada dos meses o más (por ejemplo, cuando se reúne un dinero considerable para ser enviado o inclusive cuando se ponen de acuerdo varias personas de la misma familia, por ejemplo hermanos). Por lo general, se hace referencia a que las remesas son para gastos «de la casa» o medicamentos para los mayores y para «lo que necesitan» si es para los hijos que se encuentran en Paraguay. Roberto (Paraguari, 25) solía enviar 650 pesos mensuales (alrededor de US\$ 150), aunque a veces más. Se los manda a su mamá, la que destina la mitad de ese dinero a su ex mujer, para que lo utilice en las necesidades de sus hijos. Las mujeres suelen ser las administradoras de los dineros de las remesas. Entonces, el mismo entrevistado hacía referencia a que se utilizaba para «comprar pañales, fruta, yogurt, todo eso, nada más para esto, después todo leche. No compra leche, no compra verdura, todo saca la huerta. Y cuando la carne, ella compra a veces una vaca entera digamos, como te digo... La freeza, la vida económica más o menos zafa». A veces, juntan el dinero con su hermano y su hermana, el que también se utiliza «para hacer cualquier cosa en la casa, ya sea pastizal, el campo, limpiar el campo, comprar vaca, arreglar alambrado,

7 Por ejemplo, aquellos que habitan la Villa 31 de Retiro hacían referencia a que enviaban las remesas por medio de la empresa de transporte Nuestra Señora de Asunción. También suelen enviarlas desde la terminal del barrio de Once. 
cosas así, cambiar la casa, ponerle piso». Es decir, cuando los padres son mayores, el dinero a veces es utilizado para que puedan mantener el campo, porque la economía de subsistencia ni siquiera puede llegar a ese rol.

Betina nos relataba así el envío de remesas, que había comenzado antes, en situación de migración interna, al igual que muchas jóvenes:

Después cuando vinieron mis dos hermanos, se vino mi mamá, se vino mi papá, pero en realidad (ummm) se vinieron... nosotros les mandábamos plata a mamá, de lo que yo ganaba pero porque yo estaba acostumbrada a siempre mandarle plata a la familia, no... creo que la mayoría de los paraguayos hacen eso, vienen a trabajar y mandan plata a su familia. Pero después mamá me dijo que... como mamá ya tenía edad, estaba medio enferma, mi papá también, no trabajaban más, y solamente creo que vivían de lo que nosotros les mandábamos, por eso ellos dos decidieron venirse a la Argentina.

En este caso se observa cómo la migración es la estrategia para poder mantener a la familia. Juan Manuel (Guayaquil, 23) nos explicaba que enviaba dinero a sus hermanos, siendo ésta una situación menos habitual, porque si los hermanos son jóvenes y no tienen ingresos, se les invita rápidamente a migrar a Buenos Aires. El entrevistado afirmaba que le enviaba unos US\$ 75 dólares mensuales, pero a veces ellos le pedían un poco más de US\$ 100 y él le enviaba ese dinero. Fabián fue el único que indicó que pudiendo enviar dinero a su hija decidió por algunos meses no hacerlo porque «estaba haciendo inversiones en Argentina» y al mismo tiempo fue el único que decide en qué se gasta el dinero en Paraguay (su madre cuida de su hija). Las inversiones a las que hacía referencia era la construcción de cuartos para alquiler en la villa en la que vive (declaraba ganar casi US\$2.000 al año por esta vía). Por esta razón sostenía «intelectualmente somos muy bajo sobre ellos (los argentinos), pero si le damos valor al trabajo, nosotros somos $100 \%$ mejor, somos una máquina produciendo plata».

Como se indicó, en general, la comunicación con los parientes en Paraguay es fundamentalmente por teléfono celular, una vez por semana con padre, madre, hijos o con parientes más lejanos con menor frecuencia o en fechas claves como cumpleaños, día de la madre, etcétera. Algunos utilizan locutorios cuando no tienen crédito en sus teléfonos móviles. Algunos entrevistados hacían referencia que se comunicaban con vecinos ya que sus padres no tenían teléfono. En muchos casos se envían mensajes por teléfonos celulares. En ocasio- 
nes se comunican por internet. Ana relataba: «sí, sí por mensaje, siempre las llamo el día de la madre o cumpleaños, cada tanto las llamo, cada ocho les mando mensaje o cuando estoy sola, cuando me siento sola y todo eso, les mando». De esta forma, se observa que no solo se llamaba para saber cómo están los parientes o amigos, para avisar el envío de remesas, sino para remediar la situación de melancolía propia de la situación migratoria.

\section{REFLEXIONES FINALES}

Durante la juventud, tienen lugar dos transiciones claves: el paso de la educación al empleo y la autonomización del hogar de origen. Entre aquellos y aquellas que migran se presenta además un tercer recorrido: el que va del lugar de origen hacia la sociedad de destino (García Borrego, S/F). Esta situación de movilidad, muchas veces soslayada en los estudios de juventud, hace preciso incorporar el análisis de las condiciones de vida en el país de origen, para luego abordar el estudio de la situación educativa, laboral y de autonomía de vivienda de la juventud migrante en el país de destino.

La información sobre las condiciones de vida en el Paraguay da cuenta de que las transiciones juveniles se realizan en un contexto de escasez de oportunidades de ingresos y de temprana asunción de responsabilidades de producción y reproducción familiar. En este escenario, la búsqueda de nuevas opciones aparece mediada por la posibilidad de la movilidad trasnacional. A ello se le suma la existencia de consolidadas redes en el corredor paraguayo-argentino, las cuales dan contención material y psicológica durante el viaje, la llegada y el asentamiento en la sociedad de destino. Estos factores dan forma al marco en el que muchos jóvenes paraguayos y paraguayas dejan su hogar familiar y emigran de forma individual en busca de mejores posibilidades de vida, trabajo y consumo cultural.

La perdurabilidad y magnitud de los flujos, así como la latencia de la movilidad como alternativa para un futuro mejor, hacen que la migración no sea un acontecimiento percibido como excepcional. La información relevada mediante entrevistas da cuenta de la naturalización del fenómeno migratorio, de su vigencia y de la forma que adquiere en nuestros días gracias al avance de los dispositivos de comunicación. Las oportunidades laborales pueden comunicarse de manera inmediata a través de teléfonos celulares y redes sociales, lo cual suma un componente de mayor indeterminación a los proyectos migratorios, 
un rasgo característico de la forma de vida de las juventudes contemporáneas. En efecto, un conjunto de investigaciones han señalado que las modificaciones en la percepción del tiempo, la menor estructuración de los recurridos transicionales, así como la reversibilidad de las decisiones vitales con hechos cotidianos entre los/las jóvenes de distintos países y grupos sociales (Machado Pais, 2004; Leccardi, 2005).

Frente al análisis de las distintas formas de transitar la juventud, los estudios del campo han señalado la vigencia de «regímenes de transición» diferenciados (Walther, 2006). Las divergencias son relativas a las efectivas oportunidades de actividad que las sociedades brindan a la juventud durante este período transicional. Los regímenes pueden ser mayormente estatalistas - entre los que se encuentran las sociedades con mayor grado de protección social - o primordialmente privados - entre los que se encuentran aquellos en donde la emancipación es sostenida de manera principal por los grupos familiares-. El análisis de la situación social de los/as jóvenes migrantes hace suponer que la movilidad representa una de las opciones que los grupos familiares extendidos tienen a su disposición para ofrecer, frente a la ausencia de oportunidades en la sociedad de origen. Como una expresión propiamente latinoamericana de aquellos regímenes transicionales donde queda por parte de las familias sostener las actividades propias a la emancipación juvenil.

De esta forma, entre la juventud paraguaya de menores recursos la migración se posiciona como una de las opciones vigentes durante la transición hacia la adultez. Esta transición, que se presenta de manera precoz en la asunción de tareas laborales a edades tempranas, adquiere una nueva dinámica, mediante una «invitación» familiar a partir de una oportunidad de trabajo mejor remunerada. Justo con esta invitación, se procede entonces al inicio de una búsqueda relativa a la conformación de un espacio propio, el cual se consolida en la sociedad de destino mediante el alquiler de una pieza o la construcción de una vivienda en algún barrio del conurbano, luego de algunas moradas coyunturales proporcionadas por los grupos familiares ampliados que dan acogida.

Frente a estos recorridos, hemos visto, el posicionamiento de género atraviesa y define los recursos que utilizan los y las jóvenes en el logro de su emancipación. En el proceso migratorio se combinan diferencias y desigualdades de género, tanto en los contextos de salida como en los de recepción. En el contexto de origen, se ha comprobado la discriminación que sufren las mujeres en el mundo laboral, la cual 
se refleja en una menor participación en el mercado de trabajo, con su correlato en la asunción de tareas del hogar. En esta dirección, la elevada y temprana fecundidad de las jóvenes en Paraguay, a su vez, conlleva comprobadas dificultades de conciliar tareas reproductivas al interior de los hogares y familias con la vida laboral. Estas diferencias, en combinación con la demanda en Argentina de mano de obra en empleos de cuidados personales - empleo doméstico, principalmente, y cuidado de personas-, típicamente ocupados por mujeres, y las mejores remuneraciones, ayudan a comprender en parte la creciente participación de las mujeres en las corrientes migratorias y su centralidad, política y teórica, en las migraciones contemporáneas.

La migración expresa las corrientes de cambio y reproducción social propias a la condición juvenil. Como parte de la tensión permanente entre dependencia y autonomía familiar, las muchachas parecen encontrar una mayor libertad en relación a sus padres a través de la migración, a pesar del carácter doméstico de su inserción laboral y social en el lugar de destino. Lo cual se transforma en un proceso de «empoderamiento» inexorable, y mayormente irreversible. La migración joven masculina expresa, en cambio, la continuidad de un patrón de género que vincula al hombre con el empleo y la provisión económica, en tanto tarea vital hegemónica. Los espacios de trabajo en donde los migrantes se integran se hallan localizados en un segmento particular del mercado de trabajo (por ejemplo, la construcción), en donde se mantiene la hegemonía masculina, en el marco de la ausencia de derechos laborales asociados a las tareas reproductivas y familiares. Entre ambos, la lógica de la doble jornada femenina y las tensiones propias a los grupos familiares ubican a los jóvenes de ambos sexos dentro de un gran espacio compartido con las nuevas generaciones en la Argentina contemporánea.

BUENOS AIRES (ARGENTINA), JUNIO DE 2012

RECIBIDO: AGOSTO DE 2012 ACEPTADO: NOVIEMBRE DE 2012 


\section{REFERENCIAS BIBLIOGRÁFICAS}

CERRUTTI, MARCELA (2009a): «Gender and Intra-regional Migration in South America». Human Development Reports Research Papers 2009/12, United Nations Development Programme.

(2009b): «Situación de las poblaciones de inmigrantes en la Argentina». Serie de Documentos de la Dirección Nacional de Población. Dirección Nacional de Población, Ministerio del Interior, Buenos Aires.

— y EMILIO PARRADO (2006): «Migración de Paraguay a la Argentina: género, trabajo y familia en contextos de origen diferenciados». En ALEJANDRO GRIMSON y ELIZABETH JELIN (compiladores): Migraciones regionales hacia la Argentina. Diferencia, desigualdad y derechos. Buenos Aires: Prometeo.

CEPAL/OIJ (2007): La juventud en iberoamérica. Tendencias y urgencias. Santiago: CEPAL.

CRAVINO, MARÍA CRISTINA (2008): Vivir en la villa. Relatos, trayectorias y estrategias habitacionales. Los Polvorines: Ediciones UNGS. (2006): Las villas de la ciudad. Mercado e informalidad urbana. Los Polvorines: Ediciones UNGS.

HALPERN, GERARDO (2009): «La diáspora y el Paraguay territorial». MEM, Revista Miradas en Movimiento. Volumen 1.

GARCÍA BORREGO, IGNACIO (S/F): «Jóvenes migrantes y sociedades en tránsito». Disponible en: http://e-archivo.uc3m.es.

LECCARDI, CARMEN (2005): «Facing uncertainty: temporality and biographies in the new century». YOUNG, Young Nordic Journal of Youth Research.

MACHADO PAIS, JOSÉ (2004): «Los bailes de la memoria: cuando el futuro es incierto». Revista Jóvenes, Año 8, №20. México: IMJ.

MIRANDA, ANA (2007): La nueva condición joven: educación, desigualdad, empleo. Buenos Aires: Fundación Octubre de Trabajadores de Edificios.

MOLGAT, MARC (2008): «Juventud y movilidad en Canadá: migración interna, inmigración e implicaciones de políticas públicas». En R. BENDIT, M. HAHN y A. MIRANDA (editores): Los jóvenes y el futuro: procesos de inclusión social y patrones de vulnerabilidad en un mundo globalizado. Buenos Aires: Prometeo Libros.

PORTES, ALEJANDRO (2011): «Migración y desarrollo: un intento de conciliar perspectivas opuestas». Revista Nueva Sociedad No233. Caracas: Nueva Sociedad.

PNUD, Programa de Naciones Unidas para el Desarrollo (2009): Ampliando horizontes: emigración internacional paraguaya. Asunción: PNUD, UNICEF, OIM Y UNFPA.

SANCHÍs, NORMA y CORINA RODRÍGUEZ ENRÍQUEZ (coordinadoras) (2011): El papel de las migrantes paraguayas en la provisión de cuidados en Argentina. Buenos Aires: ONU Mujeres. 
SARAVI, GONZALO (2009): «Juventud y sentidos de pertenencia en América Latina: causas y riesgos de la fragmentación social». Revista de la CEPAL $\mathrm{N}^{\circ}$ 98. Santiago: CEPAL.

TEXIDÓ, EZEQUIEL y otros (2003): «Migraciones laborales en sudamérica: el mercosur ampliado». Estudios sobre Migraciones Internacionales $\mathrm{N}^{\circ} 63$. Ginebra: OIT.

WALTHER, ANDREAS (2006): «Regimes of youth transitions: choice, flexibility and security in young people's experiences across different European context». YOUNG, Nordic Journal of Youth Research 14. 